\title{
Editorial
}

\section{The current "next step" in neuropsychiatric disorders studies}

Tremendous advances have occurred in molecular biology over the past decade. Not least of these has been the complete sequencing of human genome. We now know there are approximately 60,000 genes that are encoded on our 46 chromosomes. One third of these genes are highly enriched within the central nervous system and mutations are thought to contribute to a number of developmental and psychiatric disorders. The functions of the vast majority of the proteins expressed by these genes, however, remain to be determined. In fact, this lack of clarity has made the new era be named as "functional genomics", and many laboratories are devoting their research efforts to determining what the functions of these proteins are within the brain.

One approach that a number of investigators have taken is to remove the gene of interest from the genome and then study the phenotype of the animal in the absence of that particular gene. These animals are termed "knockouts" and many hundreds have been produced to date. One example was the recent knockout of the dopamine transporter gene. The protein is normally found at the synaptic cleft and rapidly removes the neurotransmitter dopamine from the synaptic area so that a subsequent signal can be passed across the synapse. These mice phenotype was of some interest as the animals were hyperactive and responded to stimulant medication with a decrease in their hyperactivity. ${ }^{1}$

Investigators have recently been studying another knockout mouse that has been proposed as a good animal model for autism, a pervasive developmental disorder of childhood. The knockout of the oxytocin gene in mice produces a deficit in social memory, a key clinical feature of autistic children. Intriguingly, if the missing neuropeptide is administered before conducting the experiment, the mice appear to recovery some of their social memory ability. ${ }^{2}$ The model suggests that oxytocin-specific receptors expressed in the medial amygdala may prove essential for certain aspects of social memory tasks. The model may be helpful in clarifying this behavior's functional anatomy.

Occasionally, mutations occur naturally in mice. The phenotype of some of these mice has proven particularly interesting to neuroscientists interested in the development of the nervous system. An example was a mouse which had a curious inside-out reversal of the normal laminar pattern of the cortical plate. The identification of the gene responsible for this mutation was only possible after the recent advances made in molecular biology that have allowed investigators to isolate and characterize specific genes. The gene responsible for the particular phenotype in the reeler mouse has been recently identified. The mutation lies in a gene that encodes for the reelin protein, which is secreted by specific neurons in the cortex and is thought to be a signal that guides migrating neurons to their proper cortical lamina. Mutation of this gene leads to specific disruption of neuronal migration during cortical development. ${ }^{3}$

Some of the newer techniques are in fact quite powerful in clarifying the intracellular signaling events that may be involved in more complex neuropsychiatric disorders. A group of researchers generated an animal model in which mice express a form of cholera toxin inside neurons. ${ }^{4}$ This toxin is not normally found in the brain, but was targeted to particular sets of neurons to disrupt their normal signaling pathways. The mice in question showed repetitive behaviors, such as repetitive biting and grooming, that were reminiscent of obsessive-compulsive-like behaviors. The investigators then proceeded to determine that the neurons of these transgenic mice have a chronic activation of a critical intracellular second messenger, cAMP, and the overproduction of this molecule appeared to be under the control of dopamine D1-receptor. Knowing more about this receptor and where it is expressed and how it may be upregulated may give hints about possible etiological mechanisms for obsessive-compulsive disorder. The dopamine D1-receptor has a spatially restricted expression, limited to specific cortical regions including the piriform and somatoform cortex. Based on increased cAMP levels, the animal model has lent support to the idea that cortical and limbic neurons may become chronically activated in this disorder. Their abnormal activation would induce an increase in the major excitatory glutamatergic output from the cortex to the striatum causing repetitive behaviors.

It is well appreciated that there is a relationship between the central nervous system and the immune system. The 
body's normal response is to produce antibodies to fight particular infections. In general, these immune responses to infection proceed normally, and the individual has a quick recovery. But occasionally the antibody response that is mounted to fight the infection has an abnormal functioning, and antibodies attack self-proteins. This abnormal response leads to an autoimmune disorder. Autoimmune responses have been suggested as a possible etiology for some neuropsychiatric disorders, including Tourette's syndrome, obsessive-compulsive disorder, and Sydenham's disorders. Several laboratories have attempted to generate animal models by infusing sera from patients having the disorders. It is hypothesized that these sera contain putative autoantibodies. If autoantibodies are introduced into the central nervous system, they may be able to disrupt the normal functioning of neurons, inducing some of the illness symptoms. By infusing Tourette's syndrome patients sera with high levels of antineural autoantibodies, a group of researchers ob- served that these sera induced more repetitive behaviors in these rats than the observed in other animals which were infused with sera from TS patients who did not have autoantibodies, or sera from normal controls. ${ }^{5}$

In short, taking advantage of molecular biology advances allowed the development of several animal models recently. Based on these models, etiological and pathophysiological hypotheses of different neuropsychiatric disorders have been tested. This approach's potential seems to be limited only by our ethological understanding and its translation into human behavior, which several groups have been studying.

Paul J. Lombroso

Child Study Center, Yale University Medical School

Marcos T. Mercadante

Programa de Pós-Graduação em Distúrbios do Desenvolvimento da Universidade Presbiteriana Mackenzie e Protoc do IPq/HC/FMUSP

\section{References}

1. Gainetdinov RR, Caron MG. An animal model of attention deficit hyperactivity disorder. Mol Med Today 2000;6:43-4.

2. Ferguson JN, Aldag JM, Insel TR, Young LJ. Oxytocin in the medial amygdala is essential for social recognition in the mouse. J Neurosci 2001;21(20):8278-85.

3. Rice DS, Curran T. Role of the reelin signaling pathway in central nervous system development. Annu Rev Neurosci 2001;24:1005-39.

4. Campbell KM, de Lecea L, Severynse DM, Caroon MG, McGrath MJ, Sparber SB et al. OCD-Like behaviors caused by a neuropotentiating transgene targeted to cortical and limbic D1+ neurons. J Neurosci 1999;19(12):5044-53

5. Taylor J, Morshed S, Parven S, Mercadante MT, Scahill L, Peterson BS et al. An animal model of Tourette's syndrome. Am J Psych 2002;159:657-60. 